\title{
Management of patients with normal-pressure hydrocephalus by using lumboperitoneal shunt system with the Codman Hakim programmable valve
}

\section{Chia-Cheng Chang, M.D., Nobumasa Kuwana, M.D., and Susumu Ito, M.D.}

Department of Neurosurgery, Yokohama Minami Kyosai Hospital, Yokohama, Japan

Thirty-two patients with normal-pressure hydrocephalus were managed using the lumboperitoneal (LP) shunt system in which the Codman Hakim programmable valve was utilized. The initial opening pressure was set at $100 \mathrm{~mm} \mathrm{H}_{2} \mathrm{O}$ in three cases, $80 \mathrm{~mm} \mathrm{H}_{2} \mathrm{O}$ in two, $70 \mathrm{~mm} \mathrm{H}_{2} \mathrm{O}$ in three cases, $50 \mathrm{~mm} \mathrm{H}_{2} \mathrm{O}$ in 23, and $30 \mathrm{~mm} \mathrm{H}_{2} \mathrm{O}$ in one case (mean pressure $57.8 \mathrm{~mm} \mathrm{H}_{2} \mathrm{O}$ ). In 12 patients the valve pressure required reprogramming. The final valve pressure was $30 \mathrm{~mm} \mathrm{H}_{2} \mathrm{O}$ in four cases, $50 \mathrm{~mm} \mathrm{H}_{2} \mathrm{O}$ in 19,70 mm $\mathrm{H}_{2} \mathrm{O}$ in three, $80 \mathrm{~mm} \mathrm{H}_{2} \mathrm{O}$ in one, $100 \mathrm{~mm} \mathrm{H}_{2} \mathrm{O}$ in two, $130 \mathrm{~mm} \mathrm{H}_{2} \mathrm{O}$ in one, $140 \mathrm{~mm}_{2} \mathrm{O}$ in one, and $170 \mathrm{~mm} \mathrm{H}_{2} \mathrm{O}$ in one case (mean $62.5 \mathrm{~mm} \mathrm{H}_{2} \mathrm{O}$ ). Lumboperitoneal shuntography was performed in five patients by injection of contrast medium into the prechamber. In two patients a shunt obstruction developed between the valve and the lumbar catheter. Complications occurred in eight patients. Low-pressure symptoms such as headache and vomiting were observed in four patients but disappeared after increasing the valve pressure. One patient, whose shunt pressure was set at $50 \mathrm{~mm} \mathrm{H}_{2} \mathrm{O}$ developed a slight asymptomatic subdural effusion, which resolved after increasing the valve pressure to $100 \mathrm{~mm}$ $\mathrm{H}_{2} \mathrm{O}$. Convulsions developed in two aged patients but were easily controlled with anticonvulsant medication. Surgery-related wound infection was observed in one patient.

The major advantage in the use of this valve is the ability to modify the pressure noninvasively. Another important advantage of the LP shunt system in which this valve is incorporated is the lower pressure of $30 \mathrm{~mm} \mathrm{H}_{2} \mathrm{O}$ that can improve symptoms caused by underdrainage of cerebrospinal fluid.

Key Words * normal-pressure hydrocephalus * lumboperitoneal shunt * programmable valve

The simplified percutaneous technique for the placement of a lumboperitoneal (LP) shunt is accepted as a useful procedure for the treatment of normal-pressure hydrocephalus (NPH). [3,4] There are three types of one-piece LP shunt systems with different opening pressures, which are used in the treatment of patients with communicating hydrocephalus: high-, medium-, and low-pressure systems. A high- or medium-pressure system is selected if the patient is expected to be able to walk postoperatively. If the patient is expected to remain bedridden, a low- or medium-pressure system is used. However, selection 
of the system with the correct pressure is sometimes difficult because the rate of cerebrospinal fluid (CSF) production and the pressure-flow curve are not precisely known in individual patients. Because underdrainage or overdrainage of CSF sometimes occurs, the shunt must be revised to another type with a different pressure setting.

The Codman Hakim programmable valve was developed by Drs. Carlos Hakim and Saloman Hakim. The pressure of the valve can be adjusted noninvasively by using a programmer that transmits codified magnetic impulses that activate the stepper within the inlet valve housing. Eighteen different pressure settings that range from $30 \mathrm{~mm} \mathrm{H}_{2} \mathrm{O}$ to $200 \mathrm{~mm} \mathrm{H}_{2} \mathrm{O}$ in steps of $10 \mathrm{~mm} \mathrm{H}_{2} \mathrm{O}$ can be selected. Therefore, patients with symptoms caused by overdrainage or underdrainage of CSF can be managed noninvasively by reprogramming the valve pressure. Although this valve has been used in the treatment of NPH by ventriculoperitoneal (VP) shunting,[1] no experience with the pressure valve and the LP shunt system has been described.

In this report we describe our experience in using LP shunt systems in which the Codman Hakim programmable valve is incorporated for the treatment of NPH.

\section{CLINICAL MATERIAL AND METHODS}

Thirty-two patients (13 men and 19 women) ranging in age from 50 to 81 years (mean 66.7 years) with NPH were treated at our hospital between August 1996 and February 1999. The cause of NPH was subarachnoid hemorrhage in 18 patients, trauma in seven, intraparenchymal hemorrhage in one, and of idiopathic origin in six patients. All patients underwent placement of the LP shunt system in which the Codman Hakim programmable valve was used. Shunt systems had been previously implanted in six patients.

The shunt system consisted of three parts: a K-type lumbar catheter (Silascon lumbar subarachnoid catheter; Kaneka Medix Co., Tokyo, Japan), a Codman Hakim valve (Codman, a Johnson \& Johnson Co., Raynham, MA), and a Codman-Medos peritoneal catheter. The lumbar catheter was connected to the valve with a connector (Kaneka Medix Co.) in which the diameter was different at each end, because the diameter of the lumbar catheter was different from that of the inlet tube of the Codman Hakim valve. The peritoneal tube was directly connected to the valve and inserted into the abdominal cavity via a pararectal laparotomy. The valve was positioned subcutaneously at the front of the abdomen so that the reprogramming unit could be placed accurately on the valve (Fig. 1). The initial opening pressures were chosen principally according to the CSF pressure that was measured via lumbar puncture with the patient in the lateral recumbent position. When the CSF pressure was $150 \mathrm{~mm} \mathrm{H}_{2} \mathrm{O}$ or higher, the pressure of the valve was set at $100 \mathrm{~mm} \mathrm{H}_{2} \mathrm{O}$. The pressure of the valve was set at 50, 70, or $80 \mathrm{~mm} \mathrm{H}_{2} \mathrm{O}$, when CSF pressure was less than $150 \mathrm{~mm} \mathrm{H}_{2} \mathrm{O}$. If the patient was expected to remain bedridden, the pressure of the valve was set at $30 \mathrm{~mm} \mathrm{H}_{2} \mathrm{O}$. 


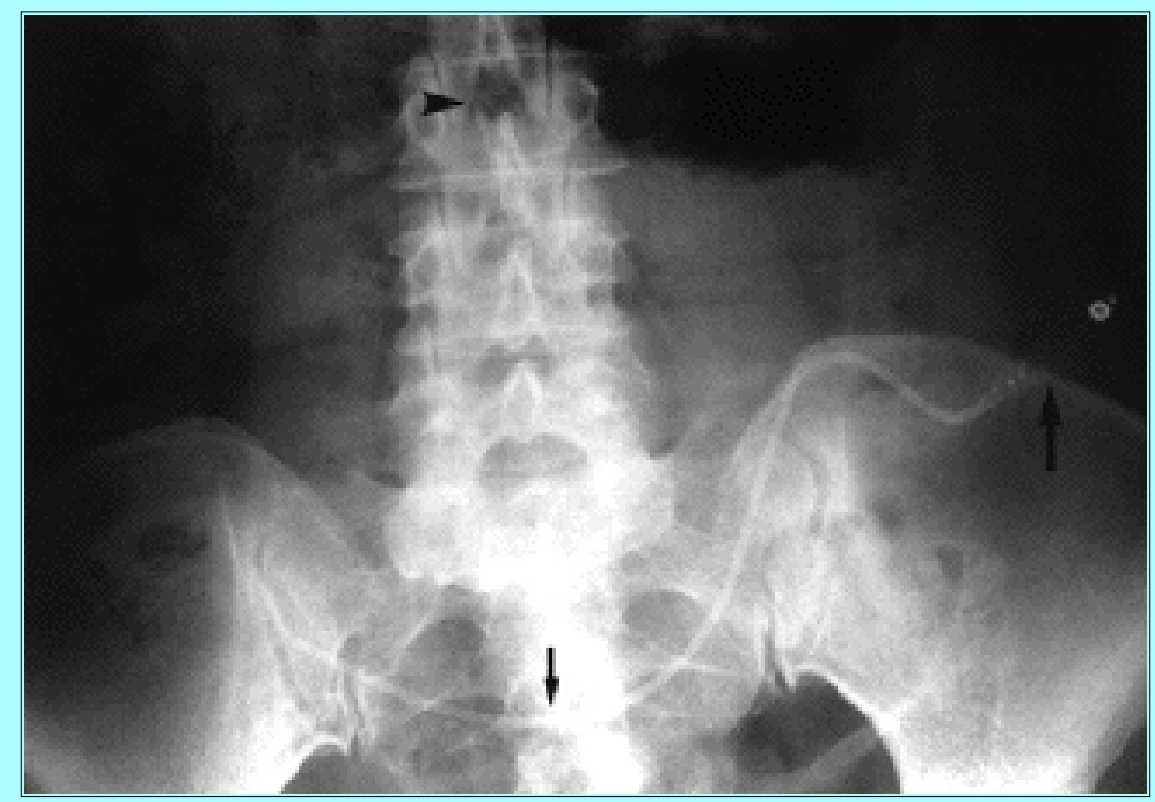

Fig. 1. Radiograph of the lower abdomen demonstrating the LP shunt system in which the Codman Hakim programmable valve was used. The lumbar catheter (arrowhead), the peritoneal tube (small arrow), and the valve (large arrow) can be recognized.

The upper part of the patient's body was kept horizontal or inclined at a 15š angle on the day of operation and gradually brought to the vertical position. Patients were allowed to sit or stand 1 week later. If symptoms or complications due to overdrainage of CSF appeared, such as headache and vomiting or subdural effusion, the pressure of the valve was increased. If symptoms did not improve and shunt dysfunction was suspected, the patency of the shunt was checked by performing shuntography of the prechamber between the valve and lumbar catheter. If underdrainage of CSF was suspected, the pressure of the valve was slowly lowered. Reprogramming of valve pressure, was accomplished by placing the reprogramming unit over the valve and resetting the pressure, using fluoroscopic guidance (Fig. 2).

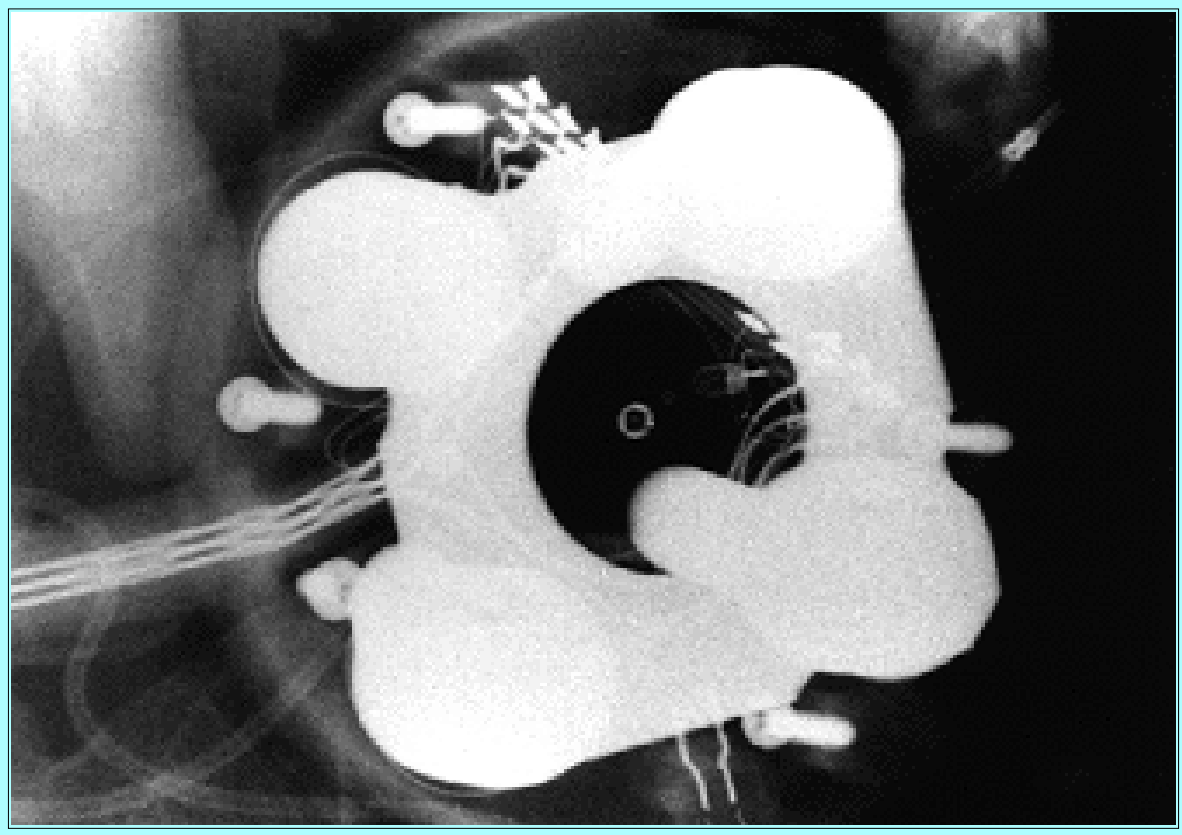

Fig. 2. Fluoroscopic image showing the reprogramming unit placed over the valve and reprogramming of the opening pressure achieved using fluoroscopic guidance. 
Thirty of the 32 patients improved after surgery, but two patients did not. The initial opening valve pressure was set at $100 \mathrm{~mm} \mathrm{H}_{2} \mathrm{O}$ in three, $80 \mathrm{~mm} \mathrm{H}_{2} \mathrm{O}$ in two, $70 \mathrm{~mm} \mathrm{H}_{2} \mathrm{O}$ in three, $50 \mathrm{~mm} \mathrm{H}_{2} \mathrm{O}$ in 23, and $30 \mathrm{~mm} \mathrm{H}_{2} \mathrm{O}$ in one patient (mean $57.8 \mathrm{~mm} \mathrm{H}_{2} \mathrm{O}$ ). In 12 patients the valve pressure required reprogramming, which was repeated one to three times. The final valve pressure was $30 \mathrm{~mm} \mathrm{H}_{2} \mathrm{O}$ in four, $50 \mathrm{~mm} \mathrm{H}_{2} \mathrm{O}$ in $19,70 \mathrm{~mm} \mathrm{H}_{2} \mathrm{O}$ in three, $80 \mathrm{~mm} \mathrm{H}_{2} \mathrm{O}$ in one, $100 \mathrm{~mm} \mathrm{H}_{2} \mathrm{O}$ in two, $130 \mathrm{~mm} \mathrm{H}_{2} \mathrm{O}$ in one, $140 \mathrm{~mm} \mathrm{H}_{2} \mathrm{O}$ in one, and $170 \mathrm{~mm} \mathrm{H}_{2} \mathrm{O}$ in one patient (mean pressure $62.5 \mathrm{~mm} \mathrm{H}_{2} \mathrm{O}$ ).

Because of underdrainage of CSF, the valve pressure was lowered in seven patients whose symptoms improved after reprogramming. The valves were reprogrammed from 100 to $70 \mathrm{~mm} \mathrm{H}_{2} \mathrm{O}$ in one, from 100 to $50 \mathrm{~mm} \mathrm{H}_{2} \mathrm{O}$ in one, from 70 to $50 \mathrm{~mm} \mathrm{H}_{2} \mathrm{O}$ in two, and from 50 to $30 \mathrm{~mm} \mathrm{H}_{2} \mathrm{O}$ in three patients. In five patients who developed low-pressure syndrome due to overdrainage, valve pressures were increased from 50 to $70 \mathrm{~mm} \mathrm{H}_{2} \mathrm{O}$, from 50 to $100 \mathrm{~mm} \mathrm{H}_{2} \mathrm{O}$, from 50 to $140 \mathrm{~mm} \mathrm{H}_{2} \mathrm{O}$, from 50 to 170 $\mathrm{mm} \mathrm{H}_{2} \mathrm{O}$, and from 80 to $130 \mathrm{~mm} \mathrm{H}_{2} \mathrm{O}$ in one case each, respectively, after which the symptoms disappeared.

Lumboperitoneal shuntography was performed in five patients by injection of $180 \mathrm{mgI} / \mathrm{ml}$ of iohexol into the prechamber, and shunt obstruction between the valve and the lumbar catheter was revealed in two patients, which was confirmed at operation for shunt revision.

Complications occurred in eight patients. The low-pressure symptoms such as headache and vomiting observed in four patients disappeared after increasing the valve pressure. One patient, whose shunt pressure was set at $50 \mathrm{~mm} \mathrm{H}_{2} \mathrm{O}$, developed a slight asymptomatic subdural effusion that resolved after increasing the valve pressure to $100 \mathrm{~mm} \mathrm{H}_{2} \mathrm{O}$. Convulsions occurred in two aged patients but were easily controlled with anticonvulsant medication. Surgery-related wound infection was observed in one patient 4 months after surgery. The valve was partly located under the operative wound, which was compressed by the belt of the trousers. The shunt system was removed, and shunt revision was performed 3 months later.

\section{ILLUSTRATIVE CASES}

We describe two illustrative cases to demonstrate the potential of the LP shunt system when combined with the Codman Hakim programmable valve.

\section{Case 1}

This 81-year-old man presented with dementia, urinary incontinence, and ataxic gait and was diagnosed as having idiopathic NPH. He underwent surgery in which the LP programmable shunt system was used, at a setting of $50 \mathrm{~mm} \mathrm{H}_{2} \mathrm{O}$. Lumboperitoneal shuntography was performed because the patient's symptoms had not improved at 3-week follow-up examination; shunt malfunction was suspected. Because the shunt system was shown to be patent, underdrainage of CSF was suspected. The valve pressure was reprogrammed to $30 \mathrm{~mm} \mathrm{H}_{2} \mathrm{O}$, and clinical improvement resulted.

\section{Case 2}

This 77-year-old woman developed the complete triad of symptoms related to NPH caused by the rupture of an aneurysm. She was managed by placement of a low-pressure VP shunt, and although her walking 
improved to some extent, she required some supervision at home. Two years postoperatively, she had become bedridden, and her practical capacity for independent function had been lost completely. After VP shuntography revealed that the shunt system was patent, underdrainage of CSF was suspected, probably due to the change of intracranial pressure. An LP programmable shunt system was placed, and the valve pressure was set at $50 \mathrm{~mm} \mathrm{H}_{2} \mathrm{O}$. She improved again but required considerable support at home.

\section{DISCUSSION}

Inclusion of the Codman Hakim programmable valve in the LP shunt system avoids the need for a second operation in which a different pressure shunt unit is implanted. The pressure of the valve can be adjusted noninvasively according to the particular conditions of the patient. However, some serious shunt system-related problems remain. In pressure valves, the main problem is to preserve the proper valve function while the patient is in the lying, sitting, and standing position. The changing hydrostatic CSF pressure in the vertebral canal changes the hydrostatic pressure, requiring the valve to adjust to a wide range of pressures, from 0 to approximately $800 \mathrm{~mm} \mathrm{H}_{2} \mathrm{O}$. Historically, LP shunting was performed by implanting a single tube: the proximal end at the CSF space in the lumbar region and the distal end intraperitoneally. After some adaptation time the patients seemed to benefit from the procedure, and no symptoms of marked overdrainage were demonstrated. However, the body position-related pressure changes in LP shunts seem to play a very important role. Therefore, care must be taken when lowering the valve pressure, especially in patients with poor brain compliance, because a large reduction of intracranial pressure may induce subdural effusion or hematoma. Because patients require some time to adjust to the programmed pressure, we recommend that the valve pressure be gradually reduced by no more than $30 \mathrm{~mm} \mathrm{H}_{2} \mathrm{O}$ within 1 week, that is if the valve pressure has already been set at less than 100 $\mathrm{mm} \mathrm{H}_{2} \mathrm{O}$.

Radiography has been used to visualize the valve assembly and pressure setting of the valve. However, fluoroscopic guidance allows the reprogramming unit to be placed more accurately over the valve, reprogramming to be accomplished in one attempt, and provides consistently readable images. Fluoroscopy is also useful in the reprogramming of the Codman Hakim valve in VP shunt systems.[2]

Lumboperitoneal shuntography can be easily performed by injection of iohexol into the prechamber when shunt malfunction is suspected. If the shunt system is patent, both the intraperitoneal spread of contrast medium and filling of the lumbosacral subarachnoid space can be visualized.

When the programmable valve is positioned under the scalp in VP shunting, artifacts are caused on magnetic resonance (MR) images of the brain. In addition, the valve pressure setting may be affected by the strong magnetic field required by MR imaging. The valve seems to be less affected by MR imaging in patients in whom the LP shunt system has been implanted because of its location at the abdomen. Although we did not observe any such change in valve pressure caused by MR imaging, we confirmed the valve pressure after each MR imaging session.

Selection of the system with the correct pressure is difficult, especially in aged patients with idiopathic $\mathrm{NPH}$. The major advantage in the use of this reprogramable shunt system is clearly the ability to modify the valve pressure noninvasively. However, we would like to emphasize another advantage of the LP shunt system in which the Codman Hakim valve is incorporated: the lower pressure of $30 \mathrm{~mm} \mathrm{H}_{2} \mathrm{O}$ can improve symptoms caused by underdrainage of CSF in patients who have already been treated 


\section{References}

1. Black PM, Hakim R, Bailey NO: The use of the Codman-Medos Programmable Hakim valve in the management of patients with hydrocephalus: illustrative cases. Neurosurgery 34:1110-1113, 1994

2. Kaufman BA, Moran CJ, Schlesinger J: Fluoroscopy of programmable cerebrospinal fluid shunt valve settings. Technical illustration. J Neurosurg 86:735, 1997

3. Kuwana N, Kuwabara T, Hosoda H: A new technique for lumbar subarachnoid peritoneal shunting for communicating hydrocephalus. Yokohama Med Bull 28:3-11, 1977

4. Spetzler RF, Wilson CB, Grollmus JM: Percutaneous lumboperitoneal shunt. Technical note. J Neurosurg 43:770-773, 1975

Manuscript received March 22, 1999.

Accepted in final form August 24, 1999.

Address reprint requests to: Chia-Cheng Chang, M.D., Department of Neurosurgery, Yokohama City University School of Medicine, 3-9 Fukuura, Kanazawa-ku, Yokohama, Japan. email: nouge@yellow.med.yokohama-cu.ac.jp. 